\title{
Germanica
}

\section{Wolfgang Hilbig. Corps humain, corps social, Une esthétique de la décomposition}

Wolfgang Hilbig - Menschlicher Körper.Sozialer Körper. Eine Ästhetik der

Verwersung

\section{Marie-Hélène Quéval}

\section{OpenEdition}

\section{Journals}

Édition électronique

URL : http://journals.openedition.org/germanica/500

DOI : 10.4000/germanica.500

ISSN : 2107-0784

\section{Éditeur}

Université de Lille

\section{Édition imprimée}

Date de publication : 1 décembre 2005

Pagination : 91-106

ISBN : 2-913857-16-7

ISSN : 0984-2632

\section{Référence électronique}

Marie-Hélène Quéval, « Wolfgang Hilbig. Corps humain, corps social, Une esthétique de la

décomposition », Germanica [En ligne], 37 | 2005, mis en ligne le 07 janvier 2010, consulté le 06 octobre 2020. URL : http://journals.openedition.org/germanica/500 ; DOI : https://doi.org/10.4000/germanica. 500

Ce document a été généré automatiquement le 6 octobre 2020.

(c) Tous droits réservés 


\title{
Wolfgang Hilbig. Corps humain, corps social, Une esthétique de la décomposition
}

\author{
Wolfgang Hilbig - Menschlicher Körper.Sozialer Körper. Eine Ästhetik der \\ Verwersung
}

Marie-Hélène Quéval

La germination de chaque vérité mène notre
corps au pressoir : nous pressons notre vie
chaque fois que nous méditons - un penseur
absolu serait donc un squelette qui cacherait ses
os dans la transparence des pensées.
Cioran, Le Crépuscule des pensées, in : Cuvres,
Quarto Gallimard Paris 1995, p. 355.

Ce n'est point un hasard si Wolfgang Hilbig place une citation de Cioran en exergue à son roman Une métaphore ${ }^{1}$ car cet auteur joue un rôle prépondérant dans sa formation intellectuelle. Ce qui le fascine chez ce philosophe, c'est son obsession de la mort et de l'absurdité de la condition humaine, sa peinture du corps blessé, ce désespoir qui mène au nihilisme, son obsession de «l'absence » qui est avant tout absence de sens. Comme Cioran, Hilbig a fait l'expérience d'une dictature, même si l'intolérance et la médiocrité idéologique d'une RDA déliquescente n'ont aucune commune mesure avec la folie apocalyptique du nazisme ${ }^{2}$. Depuis que le $\mathrm{XX}^{\mathrm{e}}$ congrès du parti communiste en URSS a révélé les crimes de Joseph Staline et balayé la dernière utopie socialiste, l'histoire de la RDA n'a plus été qu'une succession de crises ; 1953, 1956, 1968... chaque fois qu'un peuple se révolte afin de soulever la chape de plomb soviétique, l'Armée rouge intervient pour mettre fin au rêve. En cet univers clos, où l'oxygène se raréfie d'année en année, de crise en crise, il n'y a point de place pour la beauté.

2 L'art a perdu son innocence: impossible désormais de croire encore en la vertu régénératrice du beau comme au temps des Lumières! Impossible d'adhérer à l'expérience esthétique développée à Bitterfeld, à la peinture plutôt mièvre d'une classe 
ouvrière apaisée telle que la figure par exemple un tableau de Willi Sitte : «Ouvrier pendant la pause ${ }^{3}$ ", en tons de pastel! Comment croire encore à la vision de ce rude travailleur paisiblement plongé dans la lecture d'un livre pendant la pause, à la représentation de cet ouvrier au regard serein, au corps musculeux mais détendu, puissant et tranquille, sûr de sa force mais avide avant tout de savoir, bien à l'abri en haut de sa grue ? Sur le Prenzlauer Berg, dans ce quartier berlinois qui, adossé au mur, tombe en ruine, dans ces cours sordides et noires, derrière ces façades lépreuses dont les balcons menacent de s'écrouler, va naître, au cours des dernières décennies de la République, une esthétique de la dislocation du langage et des formes, une esthétique de la laideur dont relèvent les romans de Wolfgang Hilbig. La raison et l'harmonie des Lumières, la réconciliation du corps et de l'esprit prônée à Bitterfeld cèdent la place à la division, au dédoublement, à l'horrible, au difforme, au bizarre et à l'étrange. Les modèles sont désormais Samuel Beckett, Edgar Allan Poe, Charles Baudelaire... Inscrite dans la mouvance postmoderne ${ }^{4}$, cette esthétique de la laideur privilégie la peinture du corps humain, non plus celle du corps idéalisé comme celui du Prométhée de Füssli ${ }^{5}$, de cet athlète aux muscles harmonieux qui, même dans l'adversité, manifestait le triomphe de l'humanité sur la tyrannie divine, mais le corps humilié, pris dans la « machinerie du pouvoir qui le fouille, le désarticule et le recompose ${ }^{6}$ ».

Dans les romans de Wolfgang Hilbig, les images et les métaphores en effet ressortissent au domaine de la vie du corps. L'humour noir -, à l'en croire le seul possible en RDA, rebute par l'importance accordée aux fonctions les moins nobles de la chair pour décrire une situation immonde dans un État totalitaire. On assiste alors, par un réalisme grossier abaissant l'homme à ses plus viles fonctions, à un centrage sur ses besoins naturels les plus triviaux: l'absorption ou le rejet de nourriture, la consommation excessive d'alcool, le désir sexuel réduit à un instinct animal. Symboliquement privé de visage, le corps ne survit que par ses fonctions naturelles, le sang qui circule dans ses veines, qui bat contre ses tempes; il garde l'énergie nécessaire à sa survie, rien de plus. C'est un corps en mouvement, sans tête, sans raison pour le guider, animé par des pulsions incontrôlées... Pour Jacques Lacan ${ }^{7}$, le corps est sa propre exigence; il fonctionne comme un langage et son écriture postule l'expérience première que le sujet a de son sexe. Chez Hilbig aussi, loin d'exprimer la passion amoureuse, la sexualité se résume à une déjection solitaire. Les femmes, nombreuses pourtant, n'ont pas d'existence propre et ne sont vues que par le regard de l'homme qui les observe, les poursuit de son désir, les possède mécaniquement... Cette réduction de la vie à ses besoins les plus élémentaires entraîne la solitude de l'individu dans une masse anonyme. Et comme le corps emmure la conscience, de même le mur de Berlin enferme-t-il les citoyens de la République démocratique.

Dans l'univers concentrationnaire d'une RDA soumise à la toute-puissante Stasi, dans cette entreprise d'anéantissement de la personnalité humaine, le visage a perdu sa fonction identificatrice... Les personnages qu'imagine W., le héros écrivain, tout comme ceux de son auteur Wolfgang Hilbig, n'ont pas de visage ${ }^{8}$, car ils n'ont plus de caractère individuel ; ils n'ont pas de regard... impossible d'atteindre l'autre! Le visage reste invisible; et cette invisibilité est une remise en question du " Moi ». Comment un corps sans visage peut-il dire «moi » ou « je »? «Je » est un autre, disait Arthur Rimbaud", à 
quoi Hilbig répond «je » n'est personne; le «je » n'a pas de contours définis, mort comme l'ovale des mannequins, vide comme la feuille blanche.

Sans visage et sans nom, W. erre tout le jour dans les sous-terrains d'une ville inquiétante (unheimlich); épuisé, il se blottit en position de fotus sur une caisse de légumes qui semble, par une ironique allusion à Cioran, réduire l'existence à l'état végétatif... car l'homme ne peut, selon le philosophe roumain, qu'envier la condition des plantes et des végétaux ${ }^{10}$. "Combien j'aimerais être plante, dussé-je veiller un excrément !» dit Cioran ${ }^{11}$. Comme chez Edgar Allan Poe et dans les romans gothiques anglais, les lieux obscurs (caves, sous-terrains, tunnels, cachots) abondent chez Hilbig. Or, comment ne pas établir une connotation psychanalytique? Marie Bonaparte avait déjà constaté à propos d'Edgar Allan Poe que les caves avaient une valeur hautement symbolique et qu'elles signifiaient le ventre maternel, image que, chez Hilbig, la position fœtale du personnage et l'évocation de la couveuse pour désigner la ville de Berlin confirment en effet. La gnose et les sociétés secrètes des francs-maçons ${ }^{12}$ y voyaient également la grotte de l'initiation. Or, c'est bien dans un cachot de la Stasi que débute le chemin parcouru par W. tout au long des trois romans Une métaphore, "Moi » et Provisoire. Mais, seule importe la voie, non point l'arrivée; car, dans un monde absurde, il n'y a que mouvement sans but ${ }^{13}$. Dans sa caverne située sous l'immeuble de la Stasi, grand maitre du royaume des ombres comme le dieu qui, dans la tradition ésotérique, fait surgir l'univers de son souffle ${ }^{14}$, W. expire le temps, en proie à une somnolence méditative, recroquevillé sur un fauteuil, "ce grand responsable, ce promoteur de notre âme $\mathrm{e}^{15}$ ", un canapé d'un rouge éclatant, bourré de lettres et de papiers... messages incertains... lettres de dénonciations, rapports de la Stasi, témoignages honteux de toutes les ignominies humaines... Dans les bas-fonds sordides de Berlin, son regard ne rencontre que misère et laideur. Tel un rat, il se laisse envahir par l'odeur doucereuse, écœurante de la moisissure qui prolifère sur la pierre humide et pénètre l'obscurité ${ }^{16}$. Privé de lumière, d'air frais et d'espoir, W., à la suite de Cioran, " apprend à être perdant ${ }^{17}$ ».

6 W. ou C., cette créature informe aux noms multiples dont les initiales évoquent les odeurs pestilentielles de certains lieux secrets, se traîne, se recroqueville sous l'immeuble de la Stasi pour écouter les gloussements, les gargouillements des eaux usées dans les canalisations, veines et tubes digestifs de ce corps odieux de la Normannenstraße, corps qui, à la surface de la terre, envoie ses tentacules pour enserrer les citoyens avides de liberté. Par une métaphore significative, les chasses d'eau résonnent dans les tuyauteries, rappelant les activités abjectes de la Stasi. Pis encore, le personnage rêve que son corps lui échappe, qu'il ne rejette plus cette masse brune, grasse, poisseuse, brillante et nauséabonde issue de la digestion, mais qu'il se transforme bel et bien en elle pour glisser le long des tuyaux d'évacuation vers les égouts de la ville ${ }^{18}$... Prolongeant l'image, l'auteur choisit de surnommer l'officier de W., C. ${ }^{19}$ ou Cambert, "Wasserstein ${ }^{20}$ ", surnom qui rappelle à son tour les latrines hideuses. D'ailleurs, Feuerbach-Kesselstein-Wasserstein-Wasserschwein n'oublie pas de préciser que les documents rédigés par ses agents servent à la production de papier hygiénique, la Stasi aidant ainsi à surmonter la pénurie de ce produit en RDA, pénurie dont, par une sombre ironie, elle est d'ailleurs à l'origine vu la quantité considérable de rapports établis sur les citoyens soumis à son observation. Au cours du récit, C. se révèle être l'initiale de Cambert, un nom qui évoque certain produit de l'agriculture française au parfum peu apprécié des personnes sensibles. Et cet être, sans consistance, pâte molle et malodorante, se terre dans les couloirs du métro... écrasé par la honte, la peur, 
conscient de son abjection. Les fonctions les plus triviales du corps humain fournissent une image peu amène pour décrire les activités de la police secrète, la déchéance morale du mouchard. Le calambour scatologique donne ainsi un tour rabelaisien à l'humour plus que noir de Wolfgang Hilbig.

7 Les germes de la dissolution, la perte de l'innocence, ont été posés très tôt dans l'enfance du narrateur, ce jour de juin 1953, lorsque l'armée russe envahit la petite ville et que l'enfant dut faire face à l'armée soviétique... Les torses nus et luisants des soldats russes assoiffés... seuls êtres vivants dans les rues désertes, surgissaient des chars, dans la chaleur torride de l'été, semblables à des bêtes mythologiques, à des monstres ${ }^{21}$ de métal et de chair, altérés d'eau et de sang, centaures de la puissance étatique menaçant le frêle garçon... La catastrophe du 17 juin scelle le destin de W., un destin qui se déroule inexorablement d'un roman à l'autre, d'Une métaphore ${ }^{22}$ à « Moi » et à Provisoire. Les chars, métonymie de la dictature, laissent leur trace indélébile dans l'âme de l'enfant. Impossible de croire encore à l'harmonie du monde, impossible d'adhérer à l'univers. L'âme se replie, s'exclut et se condamne à une éternelle absence; cette expérience première a irrémédiablement détruit l'unicité originelle de l'être. Depuis ce jour de juin, W. surnommé Jakob pour la circonstance, n'est plus qu'un corps sans âme, une ombre, un somnambule... il a perdu la foi en ce rêve d'un monde meilleur que promettait le socialisme ${ }^{23}$. Désormais, le personnage se déshumanise, se sépare de son corps; et ce dernier, à la manière des gnostiques, n'est plus qu'une enveloppe vide ${ }^{24}$; l'âme l'observe de loin lorsqu'il se met à écrire pour s'affranchir du réel ${ }^{25}$ dont la violence meurtrière est symbolisée par le canon pointé vers la terre, vers l'enfant désarmé ; la réalité physique, celle que tous s'accordent à désigner comme telle, cette réalité-là n'est que violence, destruction et mort. Ce n'est point l'auteur qui prend la fuite ; comme l'enfant, il fait front ; non, c'est la réalité qui s'éloigne, se disloque et perd toute sa consistance.

Bien que, s'inspirant de l'Histoire et de l'actualité, elle souhaite avant tout illustrer la barbarie de la dictature et son entreprise d'avilissement de l'humanité, l'écriture romanesque de W. Hilbig dépasse la simple métaphore politique pour puiser dans un registre qui la rapproche de celle d'un Cioran: l'esthétisme mystique issu de la tradition chrétienne et renouant avec le thème de la faute originelle. Car, au-delà du simple contexte politique et historique des années 1980 en Europe, la situation d'enfermement dans un pays aux frontières bien gardées, derrière son mur de béton dérisoire et pourtant insurmontable, lui sert de métaphore pour « l'exil métaphysique " de l'humanité tel que le concevait Cioran.

C'est ainsi que, dans une vision surprenante, il élève la dépouille incandescente de Staline sur un macabre autel. Le rougeoiement de la putréfaction évoque la souillure primitive de la faute. Aucun rachat possible : Dieu s'est éloigné et se tait. Chez Jacques Derrida $^{26}$, un auteur qui, lui aussi, a une importance capitale pour Hilbig, la modernité se caractérise par l'absence de "l'écriture divine " et l'œuvre fonctionne comme un travestissement de l'origine, à laquelle l'écriture resterait liée. Or, l'origine, pour Hilbig aussi, c'est la mort de Dieu et de son substitut: Joseph Staline. Reprenant une image empruntée à la Gnose, Hilbig place à côté du Dieu suprême, inconnu mais si proche, ce Dieu de bonté, dont le christianisme décrit la perfection, une seconde divinité, principe 
du mal : le cadavre embaumé de Joseph Staline. Comparant ses angoisses à celles des mystiques $^{27}$ dont il parodie la langue, il crée une étrange ambiguïté entre le dieu toutpuissant des chrétiens et le dictateur géorgien. Fantôme totalitaire, la momie envahit les consciences; ses chairs corrompues symbolisent la décomposition du corps de la République socialiste et contaminent l'imagination malade d'une humanité en déroute. En perdant sa foi en Staline, le socialisme qui, comme par un culte idolâtre, en était la religion, a perdu sa raison d'être et laissé ses adeptes orphelins dans un monde dénué de sens.

10 Cette perte du sens, liée au sentiment de culpabilité, obsède W., le tourmente et lui fait fuir le sommeil régénérateur de la nuit, pour le soumettre au supplice d'une perpétuelle somnolence le jour ${ }^{28}$. Torturé par la faute, le pacte conclu avec la Stasi, W. ne trouve pas l'apaisement ; épuisé, il se jette au petit matin sur une couche encore imprégnée de sa sueur et de l'alcool qu'il a ingurgité pour provoquer enfin l'évanouissement bestial de la conscience. Le narrateur de "Moi», stigmatisé par le pacte diabolique conclu avec la Stasi, ne comprend que très tard qu'il fait lui-même l'objet d'une haute surveillance et que l'IM chargé de l'observer n'est personne d'autre que l'écrivain qu'il espionne. Car tout le monde est à la fois dissident et mouchard. Le serpent se mord la queue. Personne n'échappe au regard de Méduse. Satan ${ }^{29}$ a pris l'apparence d'un bureaucrate hypnotiseur, tentateur sans dimension mythique, incarnation de la médiocrité complice d'une dictature funeste, démon sans grandeur ni sadisme, compréhensif, intelligent et conscient de l'absurdité de sa tâche, un complice plus qu'un tortionnaire. Ici, le vice n'a pas de connotation morale; il est difformité, discordance, donc laideur; la faute originelle, est une erreur de comportement, une faiblesse de la conscience. La concupiscence, la masturbation ne participent pas du vice mais de l'ennui ; elle ne mettent pas fin à la solitude du héros. Les valeurs chrétiennes du bien et du mal ont disparu pour laisser place à la grisaille idéologique d'un État sans autre aspiration que celle de maintenir son pouvoir sur les consciences. Entre la geôle et la vie civile, aucune différence, pas de ciel ni d'enfer... ni coupables ni innocents... le mal est une maladie, le coupable ${ }^{30}$ un patient sans espoir de guérison...

11 Larve rampante, gluante et laide, W., C. ou Cambert, quel que soit son nom, ne se métamorphosera jamais : à quoi bon lutter ? Le héros se retire du monde et se perd à la recherche de ce «moi » qu'on lui refuse. Les romans de W. Hilbig décrivent une fuite en avant, une course éperdue pour échapper à la volonté totalitaire de l'État, lui opposer la subjectivité de l'artiste et conjurer les angoisses suscitées par une névrose collective. En vain ! Comme abasourdi par la catastrophe ${ }^{31}$, la signature concédée dans un moment de torpeur kafkaïenne, il ploie, cède et s'avilit à commettre les pires lâchetés. L'histoire universelle, dit Cioran, est l'histoire du mal: «Vous n'avez pas contribué à une catastrophe : vous disparaitrez sans trace ${ }^{32}$."

Demeurer abasourdi par sa propre catastrophe, incapable de penser ou d'agir, écrasé par les ténèbres glaciales, désorienté comme sous l'emprise de quelque hallucination nocturne ou abandonné comme dans les moments de remords, c'est atteindre la limite négative de la vie, la température extrême qui anéantira la toute dernière illusion. Dans ce sentiment d'épuisement se révèlera le sens véritable de l'agonie.... ${ }^{33}$

«Moi » raconte cette "catastrophe », cette longue agonie de l'individu marqué par la faute. Mais comment étouffer en soi les vestiges d'une volonté décomposée ? Seul le lourd sommeil de l'ivresse apporte un soulagement à cette douleur sournoise, sommeil de la résignation lâche, retour à la bestialité. Le corps négligé, mal nourri, mal lavé, ses 
sécrétions naturelles, son infâme sueur dont l'odeur se mêle à celle de l'alcool, tout exprime le relâchement de la volonté de vivre. Mis au ban de la petite société du Prenzlauer Berg, W. ne sort de sa cave que pour s'enivrer; il ne quitte le bar que lorsque l'alcool a enfin embrumé sa conscience pour se traîner honteusement vers sa chambre, sorte de tombe humide, sans air ni lumière. Tous le fuient; tous lui vouent une haine farouche ou pis encore un mépris qui le transforme en une ombre transparente. La faute pèse telle un fardeau insupportable ôtant à l'homme la dignité sans laquelle il descend à l'état de la bête.

13 C'est pourquoi le héros fictif de «Moi » préfère se retirer dans sa chaufferie, transpirer, les yeux clos, près de la chaudière vrombissante ou encore dormir protégé par une montagne de linge sale dans la laverie d'où il devrait espionner ses congénères. Alors, enfin seul, dans ce refuge obscur et étouffant, il se retrouve lui-même, et croit pouvoir écrire en toute quiétude, sans craindre le regard inquisiteur de la Stasi ; là est sa seule liberté. Le réel qu'il souhaite décrire n'a rien de commun avec celui que le parti lui impose, avec celui de Bitterfeld. La réalité n'est qu'illusion. La vérité se cache hors du monde, loin au fond de soi, dans le sommeil lourd de l'épuisement après un dur labeur à l'usine ou dans les brumes de l'alcool.

Lucide, W. a conscience du caractère incurable de son désespoir. Mais ce supplice doit être élargi à celui de toute une nation, à toute l'Europe même. Ce sentiment de culpabilité qui l'obsède depuis qu'il a signé son engagement comme IM de la Stasi, sert de paradigme à la faute universelle de ce pays déchiré, de ce continent disloqué. L'insomnie l'empêche d'adhérer au présent, au monde qui l'entoure tandis que l'ivresse ne lui permet que de sombrer dans un état proche de la mort. Le personnage vit dans l'attente, dans un espace temporel situé entre un passé écrasant et un avenir impossible, prisonnier d'un insupportable présent. Qu'il veille ou sommeille, il se trouve dans l'incapacité de vivre. Ainsi s'instaure une continuité entre la vie et la mort, continuité qui empêche aussi bien l'une que l'autre.

Or cet espace qui n'appartient ni aux vivants ni aux morts, antichambre des Enfers antiques où errent les ombres pitoyables des humains, où rôdent les divinités chtoniennes Hécate, la terrible magicienne, et Kora, l'épouse d'Hadès, c'est la terre meurtrie de Saxe, ce sont les villes qui tombent en ruines partout en RDA. Le héros d' Une métaphore évolue dans une nature sordide, "achérontique ${ }^{34}$ ", transformée en un cloaque industrie ${ }^{35}$, tandis que la clôture du roman présente les mines de charbon à ciel ouvert, royaume des morts au-dessus duquel croassent les corneilles ${ }^{36}$. La fumée ${ }^{37}$ des usines imprègne le monde. Il n'y a pas une rue, pas une place, pas un coin de la ville qu'elle n'enveloppe; cette fumée qui ôte au monde sa réalité et contribue à l'atmosphère fantastique du roman, trace dans l'air les signes de la mort omniprésente, mort de la conscience, mort du désir... Le paysage urbain déshumanisé sert de métaphore à la désolation générale, au vide moral, et occulte les trahisons, les mensonges, les crimes quotidiens du mouchard.

Poursuivi par les sbires d'un parti omniprésent, W. se réfugie dans les arrière-cours d'une ville fantasmagorique et labyrinthique, transformée en un gigantesque corps vivant dont les tunnels forment les entrailles et dont les fondations sont plantées dans la terre telles des pieux cruels dans le cœur de l'Europe ${ }^{38}$. Berlin est alors comparée à 
Moloch, à ce dieu ${ }^{39}$ dont Moïse avait interdit le culte parce que des prêtres barbares lui sacrifiaient les nouveaux-nés. Comme Moloch ou Baal, l'État dévore ses enfants, déchire l'Europe... et les bruits de la ville étouffent les gémissements de ses victimes tandis que ses caves se transforment en de noires catacombes ${ }^{40}$ !

Même la maternité glorifiée par les Vierges sereines de Raphaël, prend un aspect ignoble. Les poses indolentes, les rondeurs souples, bleues et roses des Madones italiennes ont fait place au corps anguleux et décharné d'une femme défigurée par les cachots de la Stasi. L'ovale parfait, les pommettes charnues sous le voile clair se sont creusées au point de former un masque grimaçant; le crâne rasé lors de son incarcération, altéré par de repoussantes plaques de calvitie, semble porter les stigmates d'une abominable maladie. Sans affection ni tendresse, la mère épuisée serre l'enfant contre son $\operatorname{sein}^{41}$. Le voile pudique a été remplacé par un peignoir crasseux, le visage tuméfié par les coups. Les langes souillés traînent sur le sol... Une horrible gélatine, sorte de purée en état de perpétuelle fermentation à la manière du désir inassouvi, de ce désir qui bouillonne en l'homme et l'anéantit, se gonfle, s'élance, s'étend et envahit l'appartement tout entier, écœurante, gluante et visqueuse... Perdant, soumis, incapable de résister, W. doit assumer la paternité d'un enfant dont il vient d'apprendre qu'il n'est pas le sien; or, en signant l'acte de reconnaissance que lui tend un officier vêtu d'un costume gris comme une ombre, d'un de ces milliers de costumes gris qui rendent les hommes anonymes et interchangeables, il se condamne, se lie inexorablement à la Stasi et conclut sa déchéance. En parfaite opposition à la peinture qu'en faisait le christianisme, la maternité est un crime ${ }^{42}$, la paternité un exécrable forfait.

Si, dans Une métaphore, le narrateur se consacre à une quête stérile de l'être et ne trouve dans Kora que le lieu, sans l'être, une trace sur le sol, sans le corps autour duquel la craie a imprimé sa marque, il se désagrège dans «Moi », dans ce roman où le «je »du sujet, semble s'affirmer avec force sur la couverture en réaction au nous massificateur de l'État socialiste, mais s'effrite dès les premières pages tandis que les guillemets le cernent pour signaler la faute qui le ronge. Les personnages ont tous des doubles; bizarrement, ils se scindent en deux identités comme si l'une représentait la part matérielle et l'autre la part spirituelle de l'homme ; et parfois, il semblerait que le corps insulte l'âme qui, divisée entre deux aspirations, se dédouble à son tour ; les couples ont des relations ambiguës, partagés entre la fascination et la répulsion, comme c'est le cas entre « Moi » et Cindy, l'amante dont le nom évoque les cendres de la mort. Le thème de la gémellité qui, dans le tarot déjà, représente la dualité de l'être humain, la division de l'androgyne primitif en un homme et une femme, signifie ici la crise de l'identité. Dans Une métaphore, tout se passe comme si le prénom de la mystérieuse Kora, l'inaccessible absente, sans corps ni chair, désignait la cwra heideggerienne ${ }^{43}$. Au bout d'une longue recherche, le narrateur découvre que le dessin dans la poussière ne désigne point le corps de la jeune fille ${ }^{44}$, mais le sien propre. La quête désespérée de l'être mène à l'absence ${ }^{45}$, à sa perte irréparable, à sa fuite au-delà du mur : à l'évidence de la vacuité des choses. Incapable de surmonter la dualité inhérente à son être, condamné à l'impuissance, à la passivité et à l'inaction dans un monde dénué de sens, dans un État destructeur, le personnage, forme creuse et décharnée, devient un spectre effrayant qui, dans son incapacité à adhérer au monde, ne travaille et n'agit que comme un automate. 
Sans nom, sans âme ni visage, ce corps réduit à une simple mécanique est en vérité à l'image d'un État sans idéal ni foi qui se maintient seulement grâce à la délation et à la menace, n'offrant comme alternative à ses citoyens que le bagne ou la fuite... Hilbig brosse un noir tableau des dernières années de la RDA tout en donnant à la métaphore politique un ton presque mystique. Par une étonnante liaison de l'imaginaire chrétien, parfois même gnostique, et de la réalité historique de la RDA, il pose le doigt sur le malaise profond de ses concitoyens : au cœur de ce monde tangible règne le mystère de l'absence (absence de Dieu, de foi, de conviction politique, érosion de la personnalité...) ; et cette absence de certitude ôte au monde sa crédibilité. Déstabilisés, l'homme et le citoyen, font face au vide terrible de l'absurde. Lorsqu'il donne à son personnage une valeur allégorique pour faire de lui l'incarnation même de la République démocratique, Hilbig semble renouer avec la tradition baroque. Pourtant, le lecteur reconnaît un autre genre aujourd'hui florissant; la faute, le rêve, l'étrange situation du personnage hésitant entre le réveil et le sommeil, son état de torpeur perpétuelle qui efface les frontières entre le réel et l'imaginaire, tout cela relève du fantastique. Peut-on pour autant classer les romans de W. Hilbig dans ce genre littéraire bien défini? Oui, si on reprend la définition de Roger Caillois dans l' Encyclopédia universalis : «Le fantastique [...] manifeste un scandale, une déchirure, une irruption insolite presque insupportable dans le monde réel.»Car ces romans décrivent bien le dérangement de l'ordre quotidien, l'angoisse générée par un monde en déséquilibre. En vérité, Hilbig renoue avec le fantastique Kafkaïen pour démontrer la fin des utopies. Comme chez Kafka, le lecteur assiste à la métamorphose d'un être humain, non point en une vermine, mais en une déjection du corps social. Hilbig trace le portrait d'une RDA moribonde, tout en s'interrogeant sur l'exil métaphysique de l'humanité depuis la mort de Dieu. Il ne condamne ni ne juge son personnage mais expose le fonctionnement de la dictature et la situation de déliquescence morale des intellectuels condamnés à se trahir sans cesse. Les éléments fantastiques de son écriture soulignent la folie, le caractère surnaturel et scandaleux, inadmissible pour la raison, de ce système politique. La Stasi, identifiée aux forces infernales, signifie l'irruption d'une réalité intolérable. La déchéance physique du personnage révèle la désintégration du caractère, la perte de l'identité individuelle dans un État totalitaire et inversement la décomposition de l'âme s'exprime dans celle de son enveloppe charnelle. Mal soigné, avec ses besoins non maîtrisés, le corps humain symbolise le corps malade de la RDA rongé par le cancer de la Stasi. La déchéance physique du héros manifeste sa déchéance morale, le corps avili, la dissolution du corps social.

\section{NOTES}

1. «Sich der Angst bedienen, nicht um die Abwesenheit zu einem Mysterium zu machen, sondern das Mysterium zur Abwesenheit.», E.M. Cioran. W. Hilbig n'indique pas la référence. 
2. Ce qui n'empêche pas Hilbig de faire le rapprochement de la Stasi et des perversions nazies : «[...] solche Geschichten seien ihm bisher nur von irgendwelchen perversen Nazis zu Ohren gekommen. », «Ich », Frankfurt am Main, S. Fischer 1993 p. 219, désormais la simple indication « Ich »désignera cette édition.

3. Willi, Sitte, Arbeitspause, 1959 in: Eugen Blume \& Roland März, Kunst in der DDR, eine Retrospektive der Nationalgalerie, Berlin 2003, p. 143.

4. Pour la définition de ce terme : cf. Jean-François Lyotard, La Condition postmoderne, Paris Les éditions de Minuit 1979.

5. Johann Heinrich Füssli, Herakles erlegt den Adler des Prometheus, (um 1781), Kunsthaus Zürich, Zürcher Kunstgesellschaft, Jahresbericht 1998, p. 68.

6. Michel Foucault, Surveiller et punir, Paris Gallimard 1975, p. 162.

7. Jacques Lacan, «Subversion du sujet et dialectique du désir dans l'inconscient freudien » in : Écrits, Seuil, p. 793.

8. « Gesichter hatten sie nicht, ihre Gesichter waren gleichsam weiße Blätter geblieben, auf deren Flächen jede Prägung noch möglich war », «Ich », p. 89-90.

9. « [...] ich habe in meinem Kopf eigentlich die Erinnerung eines anderen... oder aber, ich sei ein anderer, und ginge nur mit den Bildern meines vergangenen Lebens spazieren.", Eine Übertragung, p. 19.

10. «Rien d'étonnant, par conséquent, à ce que l'homme en arrive parfois à être jaloux d'une plante, d'une fleur. Pour vouloir vivre comme un végétal, grandir enraciné, s'épanouissant puis se fanant sous le soleil dans l'inconscience la plus parfaite, vouloir participer à la fécondité de la terre, être une expression anonyme du cours de la vie, il faut désespérer du sens de l'humanité. Pourquoi n'échangerais-je pas mon existence contre celle d'un végétal ? Je sais ce que c'est que d'être homme, d'avoir des idéaux et de vivre dans l'histoire : que puis-je encore espérer de ces réalités-là? " Cioran, Sur les cimes du désespoir, in : Euvres, Quarto Gallimard Paris 1995, p. 65. Désormais nous n'indiquerons que le nom de Cioran pour désigner cette édition.

11. Cioran, Syllogismes de l'amertume, p. 810.

12. On trouve une ironique allusion dans Une métaphore où Hilbig compare la Stasi à une loge maçonnique. "Weniger sicher vor mir waren womöglich die Angestellten der Post, eines Großbetriebs, dem man in der Republik zumindest Beihilfe zu den Aktivitäten der Loge der Sicherheitsmaurer nachsagte, immer schon war ich der Meinung, dass Kelle und Posthorn besser in den Ehrenkranz des höchsten Wappens gepasst hätten als funkenschlagendes Schmiedewerkzeug in einem leicht brennbaren Strohgebinde.» Eine Übertragung, Roman Frankfurt am Main S. Fischer 1989, p. 46. Désormais nous citerons cette édition en indiquant le titre seulement : Eine Übertragung.

13. « Wissen Sie, wir dürfen das Ziel gar nicht erreichen... » «Ich », p. 76.

14. «... er schlief ein und wachte wieder auf, und wachte, um wieder einzuschlafen, hier unten, wo die Zeit stagnierte, und es war, als entatme er aus seinen Lungen auch die Zeit. Eine Zeitlang war er der Patriarch der Unterwelt hier unten, der Alleinherrscher über ein unbekanntes halbdunkles Reich, unangefochten ruhte er, und alle Gedanken waren so ferne, ermüdende Gründe für das Leben oberhalb, dass er sie, kaum dass sie ihn berührten, leicht wieder fallen lassen konnte. », «Ich», p. 71.

15. Cioran, Syllogismes de l'amertume, p. 766.

16. «Vor mir verlor sich ein schmutziger Gang in die Finsternis, die Gemäuer, die diesen Stollen einschlossen, schienen schon in Fäulnis übergegangen, üble Flüssigkeiten rannen aus ihnen nieder und flossen an unsichtbaren Stellen ab ; sie sickerten hinunter in die Stadtkloaken, deren bitterer Dunst mich von Zeit zu Zeit überschwemmte. Dies waren die Augenblicke, in denen ich die Flucht ergriff, vom Ekel überwältigt, und mir schwor, nie wieder abzusteigen. », «Ich », p. 40.

17. "Une seule chose importe : apprendre à être perdant ", De l'inconvénient d'être né, in : CEuvres, Quarto Gallimard Paris 1995, p. 1346. 
18. «Ich», p. 250.

19. Erk Grimm op. cité plus haut identifie WC à Wiener Café, ce qui rappellerait les lectures de W. Hilbig dans le Wasserturm de Berlin, au Wiener Café et dans la Zionskirche.

20. «Die Wassersteine, bitte, nicht in den Mund nehmen! Man wusste, dass damit jene Stücke einer weißen wachsähnlichen Substanz gemeint waren, die zur Geruchstilgung in die Pissoirbecken geworfen werden ; es war ein wirklich vollbärtiger Witz, der sich fast wortwörtlich auf den Wänden aller öffentlichen Bedürfnisanstalten wiederholt. » «Ich », p. 11.

21. «Denn Jakob hatte den nicht unbegründeten Verdacht, dass die Erinnerungen an die Wirklichkeit, die ihm fehlten, auf der Seite der anderen wären. Dieser musste sich natürlich an die Panzer erinnern, die eines Tages das Polizeirevier von M. in beiden Straßen, die das Gebäude rechtwinklig tangierten, abgeschirmt hatten, die Geschützrohre drohend herabgesenkt, so dass sie auf Menschen zu zielen schienen... die Stadt lag in der Junihitze wie ausgestorben, die Sonne warf ihr Licht lautlos auf das blaue Granitpflaster nur vereinzelt tönte ein gedämpftes, metallisches Klicken durch den Ort, wenn vielleicht der Deckel eines Kochgeschirrs auf das Stahlblech eines der grün gescheckten, geschmeidigen Ungetüme gelegt wurde ; der Junge hatte sich immerhin zur Stadtmitte aufgemacht, wo er, einigermaßen beruhigt, das breite Grinsen der jungen Russen gesehen hatte, die mit der schweißüberströmten, braungebrannten Nacktheit ihrer Oberkörper aus den Gefechtstürmen ragten, von der dunklen Hitze erbarmungs-los umlodert, und die Kinder mit dem hilflosen Gegurgel ihrer zwei oder drei deutschen Wörter baten, ihnen Wasser zu beschaffen. » Eine Übertragung, Frankfurt/Main 1992, p. 221.

22. Eine Übertragung, Roman Frankfurt am Main S. Fischer 1989. «Ich», Frankfurt am Main S. Fischer 1993. Das Provisorium, Frankfurt am Main S. Fischer 1996.

23. «An diese Wirklichkeit mochte ich nicht denken, doch nicht etwa, weil ich gewillt war, mich ihr zu verweigern: im Gegenteil, ich lebte fortwährend in dem Gefühl, dass die Wirklichkeit gewillt war, sich mir zu verweigern. ", Eine Übertragung, Roman Frankfurt am Main S. Fischer 1989 , p. 222.

24. « Nicht hatte er bemerkt, dass dieser Körper nur eine verächtliche Hülle war... Niemand hatte die Verwandlung wahrgenommen. Der Junge, verborgen hinter weißen, wallenden Sommervorhängen, der auf einmal nichts anderes mehr tat, als die Feder über das Papier zu führen - er war natürlich eine Traumfigur, eine Idealgestalt - hatte wahrscheinlich überhaupt nicht bemerkt, dass Gott in diesem Jahr einem Herzversagen, infolge kurzer und schwerer Krankheit, erlegen war und dass er plötzlich allein war, ohne Gott... dass die Welt seiner Zukunft ohne Gott war, seit diesem Jahr 53, in dem der junge zwölf zu werden sich anschickte... [...] Er merkte es auch nicht, als nach einem XX. Parteitag die Mumie Gottes vorerst in einer Abstellkammer deponiert worden war [...] wenn er in seiner Nische saß, vermochte er noch mit seinem Geist umzugehen, nicht aber mit seinem Körper... während er mit dem Geist anderer Menschen überhaupt nicht mehr umzugehen wusste, dafür lediglich mit ihren Körpern. Und inzwischen nahm das Eigenleben seines Körpers seinen Lauf : er ging zur Arbeit, hüpfte und wippte in Sporthallen auf Turngeräten und Matten, schwamm in rasender Eile durch schmutziges Wasser, erlernte mühsam... sein teilnehmender Geist war völlig abwesend [...] », Eine Übertragung, p. 244.

25. « Doch, sagte der Herr, es ist nicht so schwer, tun Sie so, als wären wir gar nicht vorhanden! Nein, sagte W., Sie sind für mich wirklich nicht vorhanden !

Wunderbar! Sagte der Herr.

Sie sind nicht! Es ist nicht so, als wären Sie nicht vorhanden, sondern es ist so, dass Sie nicht vorhanden sind ! Sagte W. », «Ich », p. 101.

26. Cf. Marie-Hélène Quéval, «Wolfgang Hilbig, l'écriture de l'épuisement » in : Recherches sur le monde germanique, Regards, Approches, objets, en hommage à l'activité de direction de recherches du professeur Jean-Marie Valentin, sous la direction de M. Grimbert, M.-T. Mourey, E. Rothmund, W. Sabler, A.-M. Saint-Gilles et M. Silhouette. Paris-IV Sorbonne 2003. 
27. «[...] mit fast mystischer Zwangsläufigkeit begann ich auf dem groben Pflaster vor dem Bahnhof zu stolpern [...]. », Eine Übertragung, p. 36.

28. «-Nein - ich war bloß müde... es war morgens um drei, und ich hockte erschöpft auf den Steinstufen in einem fremden Treppenhaus. Ich war ausgelaugt, und ich sah Gespenster, - als das Treppenlicht erloschen war, hatte ich für Augenblicke über-haupt nicht mehr gewusst, wo ich war, in welchem Viertel, in welcher Strasse, in welchem Haus. Es war mir nicht gelungen, die Kellertür aufzuschließen, vor der ich saß... eine halbe Stunde hatte ich an dem Schloss gefummelt, bei dauernd wieder erlöschendem Treppenlicht, keiner der Nachschlüssel und Drahthaken, die ich bei mir trug, hatte sich als brauchbar erwiesen, und es war schon das dritte und vierte Schloss, das ich in dieser Nacht nicht hatte öffnen können... ich hatte gekämpft, alle Rücksicht fallen lassen und das Haus mit widerhallenden Rassel- und Knirschgeräuschen erfüllt. Nur mit Mühe hatte ich ein Wutgebrüll unterdrücken können, dann war mir der Ekel angekommen, und ich war zitternd auf den feuchtkalten Beton der Stufen gesunken. », «Ich », p. 19.

29. «Ich», p. 51.

30. «Der Schlaf des Patienten diente nun der Heilung von seiner Gewissensnot, er erwachte mit dem Glücksgefühl, ein wertvoller und schuldloser Mensch zu sein... » «Ich », p. 79.

31. «... ob es dabei auch um die Dinge ging, die seine Katastrophe zu nennen waren. », "Ich », p. 81.

32. Cioran, Précis de décomposition, "L'ennui des conquérants », in : CEuvres, p. 673.

33. Cioran, Épuisement et agonie, in : Euvres, p. 27.

34. " Nicht umsonst sprach ich von tödlichen Belanglosigkeiten : ich mußte fest-stellen, daß die auf den Einsatz folgenden Jahre beinahe vollständig aus meiner Erinnerung geschwunden waren... oder vielmehr darin versunken, untergegangen in einer Schlammflut, die ich acherontisch nannte. Rauch und üble Gerüche lagern weiterhin über Böden, die ich nicht mehr durchstoßen kann. » Eine Übertragung, p. 7.

35. «Allerdings widerfuhr meiner Geschichte dabei etwas Merkwürdiges: Dort, wo ich Schauplätze gewählt hatte, die außerhalb jedes für unsereinen zugänglichen Bereichs lagen Dschungel, Wüste, Felsenklippen... Seebuchten südlicher Küsten, Inseln, Häfen -, hielten die Attribute einer Landschaftsstruktur aus zweiter oder dritter Hand Einzug. Die Wälder waren nicht ursprünglich, hinter jedem Gestrüpp erwartete man, sie vom Unrat nahe gelegener Industrieanlagen verseucht $\mathrm{zu}$ sehen, an den Hafenstädten hing eine seltsam einheimische Trivialität und Schmierigkeit, die Leute, die dort verkehrten, waren keine mit Genever abgefüllten Haudegen, obwohl sie sich so aufzuführen versuchen, ihre Leidenschaften waren vordergründig, dahinter lauerte der Obskurantismus derer, die gute Mine vorm bösen Spiel zum Rassenmerkmal geworden war, ihre Ansichten waren berechnet, und ihre Berechnungen waren sonderbar kleinlich... sie kamen von den Küsten schlammiger Rinnsale her, und was der Wind durch ihre Wüste blies, war nicht Sand, sondern Asche. » Eine Übertragung, p. 224.

36. «- Macht, Nacht, Tod... Tod, Nacht, Macht ! Versetzte Waller.

- Ach, ach, ach, ach, ach... !

- Alle Krähen schienen sich nun in den Lüften zu befinden und verdunkelten das Firmament mit einem Sturm aus Geflatter, Gekreisch und tollwütigem Brause, es sah aus, als habe sich aller hier abgelagerter Unrat selbständig gemacht, sei in der Luft geflogen und zu einem infernalischen Leben erwacht. Das schwarze Rasen eines nie da gewesenen Chaos war in den Himmel gestiegen, bis weit über die Grenzen des Sichtbaren hin-aus.

- Sie sind erwacht, gellte Waller. Deutschland erwacht! Ach, ach, aach...

- Ach... ! erwiderte ich. Ach... Acheron... Acheron!

Drohend hallte meine Stimme in Richtung der in braunen Dünsten und keuchenden Nebeln versunkenen Stadt, in der die ersten spärlichen Lichter aufblitzten :

- Acheron... Acheron... Acheron !. » Eine Übertragung, p. 343. 
37. « [...] es war eine Kohle, die nicht brannte, sondern schmorte und schwelte. Und der Geruch, den sie absonderte, sank braungelb und bleischwer in die Straßen, und auch das Tageslicht drang kaum durch diesen Dunst. », «Ich », p. 121.

38. «Seit tausend Jahren - ich wusste es nicht, seit wann - war das Gestein in den Schoß der Erde gefügt, und es war unklar, wie viel tausend Jahre die Stadt noch aus-halten konnte, und bestehen bleiben konnte, mit dem unvorstellbaren Gewicht ihrer Grundmauern, die in das Herz Europas gepfählt waren. - Und alles, was wir lernen und begreifen konnten, was wir ermitteln und aufklären konnten, oben und unten und mitten in Berlin, war die Erkenntnis, dass wir enden mussten, - nicht aber der urbane Moloch Berlin... » «Ich», p. 21.

39. «Sie müssen scheitern, weil Sie den Staat vergöttern... und sie scheitern, und der vergötterte Staat mit ihnen. Und schließlich wollen sie über die Grenze, um Gott zu entkommen... und auch «Ich » will abbrechen, dachte er.) » «Ich», p. 360.

40. «Sie Katakombensau !... » «Ich», p. 16.

41. "Scheinbar war sie vor nicht langer Zeit kahl geschoren worden, nun waren die Haare in ungleichmäßigen, quer abstehenden Büscheln wieder hervorgesprossen, dazwischen waren noch kahle Stellen zu sehen, die wie Anzeichen von Gewalteinwirkung oder wie die Male einer Hautkrankheit wirkten. Mit offenbar unzulänglichen Mitteln war versucht worden, die einzelnen Haarsträhnen zu färben, es war ein missglücktes Wirrwarr unansehnlicher Schattierungen, zwischen denen weiße kahle Flecken leuchteten; auch sonst waren an Cindy die Spuren der Verunstaltungen zu erkennen : auch ihr Gesicht trug Schorfstellen, Schwellungen, die noch nicht zurückgegangen waren, zeigten sich dunkel unterlaufen. $\mathrm{W}$ wollte fragen, was mit ihr geschehen sei, doch dann bemerkte er den schier grauenerregenden Anblick, den ihre Wohnung bot. Wohin er auch blickte, war irgendein undefinierbarer Brei, von grauweißer bis gelblicher Farbe, verschüttet... oder abgelagert worden. Die Substanz war einem sonderbaren Auswurf ähnlich, und zum Teil war sie schon vertrocknet, wie Gips häufte sich der Brei in allen Winkeln des Fußbodens, Eimer, Töpfe, Pappkartons waren mit ihm gefüllt und quollen über; ein süßlicher Fäulnisgeruch beherrschte die Küche, dessen Ursprung die helle Masse sein konnte, die sich allerorts mit Gärungsflecken überzog... [...] die unverbrauchte Kindernahrung [...]» "Ich», p. 92-93.

42. " Es ist eine Schande, so einem Staat ein Kind zu machen! In diesem Staat ein Kind in die Welt setzen... das ist gar nicht wiedergutzumachen. », «Ich », p. 124.

43. Cf. Marie-Hélène Quéval, «Wolfgang Hilbig, l'écriture de l'épuisement » in : Recherches sur le monde germanique, Regards, Approches, objets, en hommage à l'activité de direction de recherches du professeur Jean-Marie Valentin, sous la direction de M. Grimbert, M.-T. Mourey, E. Rothmund, W. Sabler, A.-M. Saint-Gilles et M. Silhouette. Paris-IV Sorbonne 2003.

44. Cependant, lorsque la jeune fille est désignée par ses initiales, une autre association surgit dans l'esprit du lecteur. Elle devient en effet KL. Or, c'est juste au moment où l'auteur remplace Kora par KL qu'il évoque le nom de Dora, une tarte monstrueuses que les détenus donnent à ceux qu'ils suspectent de collaborer avec la police. Si l'on se rappelle que KL peut désigner un camp de concentration (KonzentrationsLager) et que Dora en était un situé à Buchenwald... l'initiale $\mathrm{Z}$ de Bruder Z prend elle aussi un sens étrange... Plus tard dans le roman, Dora sera le nom d'un Commandant de la Stasi... (p. 128) Hilbig s'amuse donc à troubler son lecteur par un jeu de piste plutôt déroutant, et ce n'est plus son personnage qui a un comportement « hermétique » («Ich » p. 18) mais son roman qui est, selon la tradition hermétique, un jeu de correspondances et de symboles que l'initié seul peut déchiffrer et comprendre. Car Dora, c'est aussi le cas exemplaire étudié par Sigmund Freud... L'appellation Bruder rappelle les cultes initiatiques des Francsmaçons auxquels Hilbig fait une allusion ironique au début de Une métaphore.

45. Un terme qui, comme on l'a vu, a une double connotation philosophique et se réfère à Jacques Derrida et à Cioran. 


\section{RÉSUMÉS}

La peinture du corps avili, mal soigné, alcoolisé, privé de repos et de sommeil sert de métaphore à la conscience coupable de l'écrivain paradoxalement mouchard (IM) et dissident tout à la fois. Cette métaphore a, dans un premier temps, une valeur politique et dénonce la déliquescence de la RDA dans les années 1980. Dans un deuxième temps pourtant, et cela dans l'esprit de Cioran, elle s'élargit pour traduire une angoisse métaphysique plus profonde dans un monde privé d'idéal et de repères depuis la mort de Dieu, l'expérience de l'apocalypse nazie et la découverte des crimes staliniens. L'esthétique de la laideur dans laquelle s'inscrivent les romans de W. Hilbig sert alors à récuser l'idéal de raison et d'équilibre des Lumières tout comme à remettre en cause

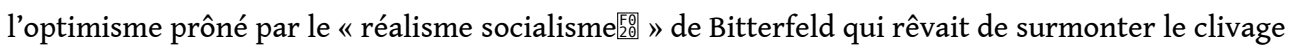
entre les intellectuels et les ouvriers.

Die Darstellung des gedemütigten, ungepflegten, alkoholisierten, unausgeruhten und vom Schlaf beraubten Körpers fungiert als Metapher für das Schuldbewusstsein des gleichzeitig als Dissidenten und IM tätigen Schriftstellers. Diese Metapher hat zuerst einen politischen Wert und zeigt die Auflösung der DDR in den 80er Jahren. Dann, als der Ausdruck der metaphysischen Angst eines vom Lebenssinn beraubten Menschen seit dem Tod Gottes, seit der nationalsozialistischen Apokalypse und der Aufdeckung der stalinistischen Verbrechen, nimmt sie, und dies ganz im Sinne Ciorans, eine metaphysische Dimension an. Die Ästhetik der Hässlichkeit dient endlich in Wolfgang Hilbigs Romanen dazu, das Vernunftideal der Aufklärung und den sozialistischen Optimismus von Bitterfeld zu widerlegen, als Walter Ulbricht davon träumte, den Klassenunterschied zwischen Arbeitern und Intellektuellen in der Kunst zu überwinden.

\section{INDEX}

Mots-clés : beauté, laideur

\section{AUTEURS}

\section{MARIE-HÉLÈNE QUÉVAL}

Université du Maine 